\title{
Approach to space-charge field description in photorefractive crystals
}

\author{
Eugenio DelRe, Alessandro Ciattoni, and Bruno Crosignani \\ Dipartimento di Fisica, Università dell'Aquila, 67010 L'Aquila, Italy, and INFM, Unità di Roma I, Rome, Italy \\ Mario Tamburrini \\ Fondazione Ugo Bordoni, Via Baldassarre Castiglione 59, 00142 Rome, Italy
}

Received May 19, 1997; revised manuscript received October 24, 1997

\begin{abstract}
Recent analytical results in the frame of photorefractive spatial-soliton propagation are exploited to derive a novel scheme for the investigation of space-charge field formation in photorefractive crystals. The procedure is specialized to describe a two-wave mixing configuration. To test our predictions, we have performed an experiment in a sample of $\mathrm{BaTiO}_{3}$. (C) 1998 Optical Society of America [S0740-3224(98)00805-4]

OCIS codes: $190.7070,160.5320,090.7330$.
\end{abstract}

\section{INTRODUCTION}

In recent decades a good deal of attention has been attracted by materials exhibiting photorefractive (PR) effect, such as barium titanate $\left(\mathrm{BaTiO}_{3}\right)$ and lithium niobate, with the main interest being focused on such applications as two-wave mixing (TWM) and holography. The standard description of the effect is based on the socalled band transport (or Kukhtarev's) model, ${ }^{1}$ which has been of continued and established success. The model, however, is highly nonlinear, and analytical solutions are approximate and limited in applicability. To date there are essentially four different approaches to the solution of the model equations (see, e.g., Refs. 2, 3, and 4), none of which are able to provide, in a straightforward manner, all of the essential relationships that tie external parameters, such as the external bias voltage and beam size and shape, to the space-charge field generated within the crystal. The most common approach is a first-harmonic linearization of the basic equations (see, e.g., Ref. 3, Chapter 3), whose solution provides an expression for the space-charge field. A second approach, particularly amenable to numerical implementation, is to solve the problem in Fourier space ${ }^{5}$ while keeping as many components as is feasible. A third approach, maybe the closest to what one may call a complete analytical solution, involves a partial linearization of the rate equation governing the effect and keeps all other nonlinearities ${ }^{6}$; however, this approach fails to provide a good description in the presence of an external-bias voltage. A fourth approach is to consider the analytical problem, solving for the ionized donor density instead of the space-charge field. ${ }^{7}$ Although numerical simulations have provided useful results (see, e.g., Refs. 8 and 9), no clear and concise full nonlinear analytical treatment is available.

The Kukhtarev model has recently been employed to describe self-trapped nonlinear propagation, leading to the formation of the so-called spatial-screening solitons, in $1+1$ and $2+1 \mathrm{D}$ (where $\mathrm{D}$ is dimensions). ${ }^{10-12}$ The analytical approach used with this model is different from the ones described above in that it deals with an optical beam of arbitrary shape resulting from the superposition of a continuum of plane waves and does not resort to preliminary linearizations. In particular, it provides a single differential equation relating the electric field $\mathbf{E}$ and the optical intensity $I$. Under suitable conditions this equation can be linearized, and an explicit solution can be found in both the $1+1 \mathrm{D}$ and the $2+1 \mathrm{D}$ cases. $^{10-12}$

In this paper we investigate the $1+1 \mathrm{D}$ case of TWM geometry, in which two spatially-limited monochromatic coherent waves interact inside a PR crystal. To this end we start directly from the differential equation for the electric field introduced in Ref. 10 and develop an appropriate iterative scheme for its solution. This allows us to recover in a simple and straightforward way the results obtained in the standard treatments, ${ }^{3,6}$ and extend them to include beam-width effects, mixing efficiency, and higher-order spatial harmonics.

To support these findings, we perform an experiment in a sample of $\mathrm{BaTiO}_{3}$. More precisely, we study the first and second space-charge field, harmonic Bragg scattering, and TWM efficiency enhancement that is due to an external biasing voltage in a high-modulation-depth situation, and we also analyze the dependence of TWM efficiency on beam size.

\section{ANALYTICAL MODEL AND RESULTS}

The PR effect is the modulation of the index of refraction of a nonlinear anisotropic crystal owing to a light-induced internal electric field $\mathbf{E}$. The index modulation is due to the Pockels effect and is proportional to the space-charge field induced by the migration of free charge carriers released by donors in illuminated areas and trapped in dark regions. The migration mechanism itself can be of vari- 
ous kinds. In this paper we study the case of only one type of charge carrier (electrons) and neglect photovoltaic effects.

The model can be represented, in steady-state conditions and restricting the analysis to one dimension, by the following set of equations ${ }^{3}$ :

$$
\begin{aligned}
\gamma N_{e} N_{d}^{+} & =(\beta+s I)\left(N_{d}-N_{d}^{+}\right), \\
(\mathrm{d} / \mathrm{d} x)(\epsilon E) & =\rho, \\
\rho & =q\left(N_{d}^{+}-N_{a}-N_{e}\right), \\
(\mathrm{d} / \mathrm{d} x) J & =0, \\
J & =q \mu N_{e} E+\mu k_{b} T(\mathrm{~d} / \mathrm{d} x) N_{e},
\end{aligned}
$$

along with the external condition

$$
V=-\int_{-l / 2}^{l / 2} E \mathrm{~d} x,
$$

where $N_{d}$ and $N_{a}$ are, respectively, the donor impurity and the acceptor impurity densities, $N_{d}^{+}$and $N_{a}^{+}$the associated ionized densities, and $N_{e}$ the conduction band electron density; in addition, $E, I, J, \rho, T$, and $V$ are, respectively, the electric field, light intensity, density of charge current, charge density, absolute temperature, and applied external voltage; $x$ is the transverse coordinate, $l$ the crystal size in this direction, and $\gamma, \beta, s, \epsilon, \mu,-q$, and $k_{b}$ the electron recombination rate, rate of thermal excitation, photoexcitation cross section, low-frequency dielectric constant, carrier mobility, electron charge, and Boltzmann's constant, respectively.

In obtaining a single differential equation, we neglect in Eq. (3) $N_{e}$ with respect to $N_{d}^{+}$and $N_{a}$, which is valid in most situations of interest $\left(N_{e} \ll N_{a} \ll N_{d}\right)$. Equation (4) implies that $J$ does not depend on $x$ and therefore assumes a constant value $J_{c}$, that is, $J=J_{c}$. Equation (2) can be used to express $N_{d}^{+}$as a function of $\mathrm{d} E / \mathrm{d} x$. Inserting this relation into Eq. (1), we are able to express $N_{e}$ as a function of $\mathrm{d} E / \mathrm{d} x$. Finally, introducing the resulting expression for $N_{e}$ into Eq. (5) and using $J=J_{c}$, we obtain, without resorting to preliminary linearizations, a differential equation for $E$ alone that reads as

$$
\left.\begin{array}{c}
\frac{q \mu(\beta+s I)\left(N_{d}-\frac{\epsilon}{q} \frac{\mathrm{d}}{\mathrm{d} x} E-N_{a}\right)}{\gamma\left(\frac{\epsilon}{q} \frac{\mathrm{d}}{\mathrm{d} x} E+N_{a}\right)} E \\
+\frac{\mathrm{d}}{\mathrm{d} x}\left[\frac{\mu k_{b} T(\beta+s I)\left(N_{d}-\frac{\epsilon}{q} \frac{\mathrm{d}}{\mathrm{d} x} E-N_{a}\right)}{\gamma\left(\frac{\epsilon}{q} \frac{\mathrm{d}}{\mathrm{d} x} E+N_{a}\right)}\right]
\end{array}\right] J_{c} .
$$

Once this equation is formally solved, the condition expressed in Eq. (6) determines the value of the current $J_{c}$. Vachss et al. ${ }^{13}$ have treated Eq. (7) in a simplified approximation in which, however, TWM cannot be described. To deal with Eq. (7), we first recast it in an appropriate dimensionless form by introducing the adimensional quantities

$$
\begin{gathered}
Y \equiv \frac{E}{E_{\mathrm{Db}}}, \quad \xi \equiv k_{\mathrm{Db}} x, \\
Q \equiv 1+\frac{I}{I_{d}}, \quad G \equiv \frac{J_{c}}{q \mu \beta_{1} E_{\mathrm{Db}}},
\end{gathered}
$$

where $E_{\mathrm{Db}}=\left(k_{b} T / q\right) k_{\mathrm{Db}}, k_{\mathrm{Db}}$ is the so-called Debye wave number defined by the expression $k_{\mathrm{Db}}^{2}$ $=\left[q^{2} N_{a} /\left(\epsilon k_{b} T N_{d}\right)\right]\left(N_{d}-N_{a}\right), I_{d}=\beta / s$ is the dark illumination, and $\beta_{1}=\beta / \gamma$. After defining $\alpha=\left(N_{d}\right.$ - $\left.N_{a}\right) / N_{a}$ and $\delta=\epsilon k_{\mathrm{Db}} E_{\mathrm{Db}} /\left(q N_{a}\right)$, we can rewrite Eq. (7) as

$$
\frac{Q Y\left(\alpha-\delta \frac{\mathrm{d}}{\mathrm{d} \xi} Y\right)}{\left(1+\delta \frac{\mathrm{d}}{\mathrm{d} \xi} Y\right)}+\frac{\mathrm{d}}{\mathrm{d} \xi}\left[Q \frac{\left(\alpha-\delta \frac{\mathrm{d}}{\mathrm{d} \xi} Y\right)}{\left(1+\delta \frac{\mathrm{d}}{\mathrm{d} \xi} Y\right)}\right]=G .
$$

We consider the standard case of $N_{a} \ll N_{d}$. This yields $\delta \cong 1, k_{\mathrm{Db}}^{2} \cong q^{2} N_{a} /\left(\epsilon k_{b} T\right)$, and $\alpha \gg 1$. Furthermore, to proceed, we neglect $\mathrm{d} Y / \mathrm{d} \xi$ with respect to $\alpha$. This last approximation is warranted both by the fact that $\alpha$ is much greater than 1 and by the fact that $\mathrm{d} Y / \mathrm{d} \xi$ is small with respect to 1 in our approach, as we assume below. With these approximations, Eq. (9) becomes

$$
\frac{Q Y}{\left(1+\frac{\mathrm{d}}{\mathrm{d} \xi} Y\right)}+\frac{\mathrm{d}}{\mathrm{d} \xi}\left[\frac{Q}{\left(1+\frac{\mathrm{d}}{\mathrm{d} \xi} Y\right)}\right]=\frac{G}{\alpha} \equiv g .
$$

It should be noted here that taking $|\mathrm{d} Y / \mathrm{d} \xi| \ll 1$ also implies some constraints on $\rho$ through Eq. (2). It can be shown that, with these restraints, $N_{d}^{+} \approx N_{a}$. This implies that, although it is generally true that $N_{e} \ll N_{a}$ $\ll N_{d}$, this does not directly authorize us to neglect $N_{e}$ in Eq. (3). An approximate condition for the simultaneous validity of the two approximations is that $N_{e} / N_{a}$ $\ll N_{a} / N_{d}$, this condition being generally true for the light intensities normally used.

To tackle Eq. (10), we introduce appropriate approximations and then employ an iterative method.

To implement our approximation scheme, we need to recall the main features of a typical TWM setup [see Fig. (1)]. Two coherent monochromatic beams, $E_{1}(x, z, t)$ $=A_{1} \exp \left(k_{1 x} x+i k_{1 z} z-i \omega t\right) \quad$ and $\quad E_{2}(x, z, t)=A_{2}$ $\times \exp \left(k_{2 x} x+i k_{2 z} z-i \omega t\right)$, are made to interfere inside the PR crystal at a certain angle $\theta$ (symmetrically, $k_{1 x}$ $=-k_{2 x}$ ) as shown, with the resulting intensity pattern having the expression

$$
I=I_{0}[1+m \cos (K x)],
$$

where $I_{0}=\left|A_{1}\right|^{2}+\left|A_{2}\right|^{2}=I_{1}+I_{2}, m=2\left|A_{1} A_{2}^{*}\right| / I_{0}$ is the modulation depth, and $K=k_{1 x}-k_{2 x}=(4 \pi /$ $\lambda) n \sin [\theta]$ is the transverse grating vector. Accordingly, the main scale of the variation of $I$ is associated with the grating vector $K$ (apart from an eventually slowly varying beam-shape factor). We must now take into account that to the dark irradiance $I_{d}=\beta / s$ introduced above one has to add a background illumination $I_{b}$ that is unavoidably present inside the crystal because of the experimental conditions (actually, the typical case is $I_{d} \ll I_{b}$ ). As a 


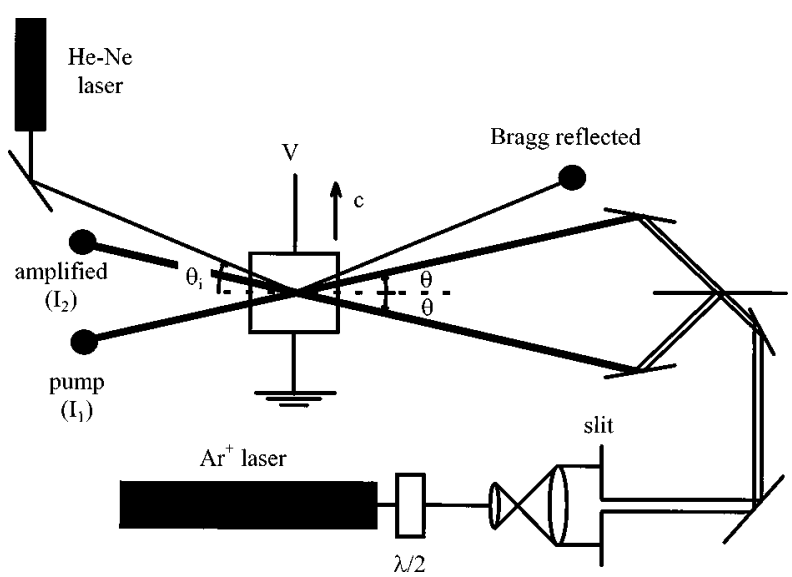

Fig. 1. Basic experimental arrangement for measuring TWM, Bragg diffraction and TWM beam size dependence.

consequence, it is convenient to modify appropriately the normalization of $I$ introduced above, so that

$$
\begin{aligned}
Q & =1+\frac{I_{0}}{I_{b}+I_{d}}[1+m \cos (\chi \xi)] \\
& =1+Q_{0}[1+m \cos (\chi \xi)] \\
& =\left(1+Q_{0}\right)\left[1+m_{1} \cos (\chi \xi)\right],
\end{aligned}
$$

where we have set $Q_{0}=I_{0} /\left(I_{b}+I_{d}\right), \chi=K / k_{\mathrm{Db}}$, and $m_{1}=m /\left(1+1 / Q_{0}\right)$.

Returning to Eq. (10) and solving for $Y$, one obtains

$$
Y=-\frac{Q^{\prime}}{Q}+\frac{g}{Q}+\frac{g}{Q} Y^{\prime}+\frac{Y^{\prime \prime}}{1+Y^{\prime}},
$$

where here the prime indicates a derivation with respect to $\xi$.

We consider Eq. (13) under the following approximations:

$$
\begin{aligned}
& \left|Y^{\prime}\right| \ll 1, \\
& \left|Y^{\prime \prime}\right| \ll\left|-\frac{Q^{\prime}}{Q}+\frac{g}{Q}+\frac{g}{Q} Y^{\prime}\right| .
\end{aligned}
$$

Conditions (14) imply that the zero-order solution of Eq. (13) is

$$
Y^{(0)}=-\frac{Q^{\prime}}{Q}+\frac{g}{Q} \equiv Y_{d}+Y_{\mathrm{dr}}
$$

while a first correction in the drift regime $\left(\left|Q^{\prime} / Q\right|\right.$ $\ll|g / Q|)$ is given by the term $(g / Q) Y^{\prime}$. To impose conditions (14), we can rewrite them consistently in the form

$$
\begin{aligned}
\left|Y^{0^{\prime}}\right| & \ll 1, \\
\left|Y^{(0)^{\prime \prime}}\right| & \ll\left|-\frac{Q^{\prime}}{Q}+\frac{g}{Q}+\frac{g}{Q} Y^{(0)^{\prime}}\right| .
\end{aligned}
$$

Equation (15) is composed of two terms. The first is essentially the diffusion field, which we indicate with $Y_{d}$; the second is the drift term, which we indicate with $Y_{\mathrm{dr}}$. Making use of the expression for $Q$ given in Eq. (12), a sufficient condition for the validity of relation (16) can be proved to be ${ }^{14}$

$$
\epsilon \ll 1, \quad\left|Y_{\mathrm{dr}}\right| \ll \frac{1}{\epsilon},
$$

where (see Appendix A)

$$
\boldsymbol{\epsilon}= \begin{cases}\frac{\chi m_{1}}{1-m_{1}} & m_{1}>\frac{1}{2} \\ \chi & m_{1}<\frac{1}{2}\end{cases}
$$

We consider now the case in which $m_{1} /\left(1-m_{1}\right) \approx 1$. In the drift regime $\left(\left|Y_{\mathrm{dr}}\right| \gg \mid Y_{d}\right)$ conditions (17) imply that Eq. (13) can be approximated to the zero order in $\epsilon$ by

$$
Y=\frac{g}{Q}+o(\epsilon)
$$

This solution describes the modulation of the crystal impedance that is due to the light intensity pattern $Q$. It completely neglects diffusion effects and fails to describe the TWM between the two writing beams introduced above because the field is in phase with the light distribution. Taking into account the terms of first order in $\epsilon$, we obtain iteratively the first-order approximate solution

$$
Y=-\frac{Q^{\prime}}{Q}+\frac{g}{Q}-\left(\frac{g}{Q}\right)^{2} \frac{Q^{\prime}}{Q}+o\left(\epsilon^{2}\right)
$$

This expression has, in addition to the zero-order drift contribution $Y_{\mathrm{dr}}$, the $Y_{d}$ contribution and a term that couples drift and diffusion [proportional to the square of the applied voltage $V$, see Eq. (21)]. The first and the last terms on the right-hand side describe possible TWM between the two beams. The last term describes the enhancement of TWM with increasing external voltage. Equation (20) therefore appears to possess all the ingredients needed for a proper description of a quite general case (within the limitations imposed by the approximations used). To give a final form to Eq. (20), we impose condition (6). The approximate expression for $g$ that we obtain, valid for solutions of both Eqs. (19) and (20), is (see Appendix B)

$$
g=\left[\frac{-V}{l E_{\mathrm{Db}}}\right] \frac{1}{\left(1-l_{b} / l\right)+\left(l_{b} / l\right) /\left[\left(1+Q_{0}\right)\left(1-m_{1}^{2}\right)^{1 / 2}\right]},
$$

where $l_{b}$ is approximately the transverse dimension of the region in which $Q$ is sensibly larger than 1 . If we assume that $V<0$, and $\left(1+Q_{0}\right)\left(1-m_{1}^{2}\right)^{1 / 2}>1$, Eq. (21) becomes

$$
g=\left[\frac{-V}{l E_{\mathrm{Db}}}\right]\left(1+Q_{0}\right)\left(1-m_{1}^{2}\right)^{1 / 2}=g_{\max }, \quad \text { for } \frac{l_{b}}{l}=1,
$$

$g=\left[\frac{-V}{l E_{\mathrm{Db}}}\right]=g_{\min }, \quad$ for $l_{b}=0$.

Eq. (21) allows us to obtain the explicit solution from Eq. (20). Condition (17) on $Y_{\mathrm{dr}}$ can be uniformly satisfied for all values of $l_{b}$ if we impose

$$
g_{\min } \ll \frac{1}{\epsilon} \frac{1-m_{1}}{\left(1-m_{1}^{2}\right)^{1 / 2}} .
$$

For the diffusive regime, in which the constant current $J_{c}$ is approximately zero $(g \cong 0)$ and $\left|Y_{\mathrm{dr}}\right| \ll\left|Y_{d}\right|$, conditions (17) imply that the lowest-order solution in $\epsilon$ is 


$$
Y=-\frac{Q^{\prime}}{Q}+o\left(\epsilon^{2}\right)
$$

The electric field worked out in Ref. 6, valid for $l_{b} / l=1$, coincides with the expression given in Eq. (15), supplemented by Eq. (22). In our approach this solution is of intermediate order in the drift regime; that is, the diffusive term $Y_{d}$ is, under conditions (17), of the same order as the first correction, with the full expression given by Eq. (20) [along with Eq. (22)]. On the other hand, in the purely diffusive regime the two expressions coincide. Furthermore, it is easy to show that for $m \ll 1$ the expression given by the standard approach (see, e.g., Ref. 3, Chapter 3) coincides with Eq. (20) to the first order in $\epsilon \cong \chi$.

\section{EXPERIMENT}

To test some of the results found in Section 2 and to give an example of the possible implementations of the novel approach, we carried out a set of experiments on a sample of standard $\mathrm{BaTiO}_{3}$. Primarily, two innovative results were tested. The first is the TWM and Bragg diffraction enhancement that is due to an external bias voltage in the high- $m$ configuration. The second is the dependence of the electric field on the (limited) size of the beams described by Eqs. (20) and (21).

To relate the results obtained in the previous section to experiments, we must recall that a static field $\mathbf{E}$ gives rise to a linear modulation of the index of the refraction tensor given by (see, e.g., Ref. 15)

$$
\Delta\left[1 / n^{2}\right]_{i j}=r_{i j k} E_{k},
$$

where $r_{i j k}$ are the components of the electro-optic tensor. In our experimental configuration, as described below, Eq. (26) is reduced to the simple form (see, e.g., Ref. 3, Chapter 4)

$$
\Delta n \cong-1 / 2 r n^{3} E,
$$

where $r$ and $n$ are the relevant electro-optic coefficient and refractive index, respectively. In addition, we recall that the Bragg diffraction efficiency $\eta_{i}$ of the grating harmonic $i(i=1,2,3, \ldots)$ is given by (see, e.g., Ref. 8)

$$
\eta_{i} \cong\left[\frac{n^{2} r k_{R}}{4 \cos \left(\theta_{i}\right)} L E_{(i)}\right]^{2}
$$

where $k_{R}$ is the wave number of the Bragg reflected light, $L$ is the thickness of the crystal in the direction of propagation of the light, $E_{(i)}$ is the space-charge-field spatialharmonic component of order $i$, and $\theta_{i}$ is its Braggmatching angle.

TWM coupling with higher harmonics in the index modulation, owing to higher harmonics in $E(i>1)$, is an extremely complicated issue (see, e.g., Ref. 4, Chapter 7) and is beyond the scope of this paper. Here we analyze only coupling between the two writing beams, this being justified both by the facts that in our conditions TWM is generally small and that higher-order components are a correction to the first harmonic. In these approximations, we take the TWM energy-coupling constant $\gamma$ as being proportional to the antisymmetric part of the first component $E_{(1)}^{a}$ of $E$, thus obtaining (see Ref. 3, Chapter 4)

$$
\gamma=\frac{\pi n^{3} r}{\lambda \cos (\theta)}\left|E_{(1)}^{a}\right|
$$

Results for TWM are given in the form of the amplifiedbeam gain ratio

$$
\frac{I_{2}(L)}{I_{2}(0)}=\frac{1+M}{1+M e^{-\gamma L}} \exp \left(-\alpha^{\prime} L\right),
$$

where $M=I_{1}(0) / I_{2}(0), \alpha^{\prime}$ is the material absorption constant, $I_{1}(0)$ and $I_{2}(0)$, respectively, are the pump and the amplified beam intensities before entering the crystal, and $I_{2}(L)$ is the value of the amplified beam intensity at the exit face.

\section{A. Experimental Setup}

The setup is based on a typical TWM or two-wave holographic geometry and is schematically illustrated in Fig. 1. A single-mode argon-ion laser emits a $\lambda=515 \mathrm{~nm}$ vertically polarized beam. A $\lambda / 2$ wave plate can be used to rotate the polarization of the beam, thus making it parallel to the plane of the optical table (parallel to the plane of the figure). The beam is expanded by means of a pair of lenses, and an adjustable slit selects the central part of the beam, thus determining its transverse dimension $\left(l_{b}\right)$. A beam splitter separates this beam into two, generating the two interfering beams $\left(\mathrm{E}_{1}\right.$ and $\left.\mathrm{E}_{2}\right)$, each of which is separately attenuated by a neutral density filter and made to impinge on a $\mathrm{BaTiO}_{3}$ crystal, thus giving the possibility of controlling the value of $m$. The $4.8^{(\mathrm{a})} \mathrm{mm}$ $\times 3.8^{(\mathrm{b})} \mathrm{mm} \times 4.7^{(\mathrm{c})} \mathrm{mm}$ crystal is cut along its principal axes and is oriented so as to have its $c$ axis parallel to the plane of the page. The geometry is entirely symmetric; that is, the two interfering beams give rise to a grating vector $K$ that is parallel to the $c$ axis. The angle between the two interfering beams outside the crystal is $2 \theta_{\text {ext }}$ $\cong 7.16^{\circ}$ (corresponding to $2 \theta \cong 2.98^{\circ}$ ). The relevant electro-optic coefficients of the sample have the measured values of $r_{33} \cong 110 \mathrm{pm} / \mathrm{V}$ and $r_{13} \cong 12 \mathrm{pm} / \mathrm{V}$, whereas the absorption coefficient has a value of $\alpha \cong 2.9 \mathrm{~cm}^{-1}$ at $\lambda=515 \mathrm{~nm}$ (a single-crystal-face reflection was taken to be $\sim 4 \%$ ). By means of gold electrodes deposited on the $c$-axis faces of the crystal, an external voltage source supplies the bias voltage $V$. The beam that experiences gain $\left(\mathrm{E}_{2}\right)$, owing to the orientation of the crystal, is detected by a silicon photodiode, and the data sampled is transferred to a personal computer. The pump beam $\left(\mathrm{E}_{1}\right)$ is monitored via a second detector, and, finally, a (extraordinarily polarized) He-Ne laser beam operating at $\lambda=632.8 \mathrm{~nm}$ and at approximately a $1-\mathrm{mW}$ power level is directed by means of an adjustable steering system on the crystal so as to be Bragg matched with the various harmonics of the grating written by the two interfering beams. Bragg reflection is detected by a third silicon photodiode, and data is again transferred to the personal computer. 


\section{B. TWM Effects in the Holographic Regime}

(High $m, \chi \ll 1$ )

When attempting to write a hologram inside a PR crystal such as $\mathrm{BaTiO}_{3}$, one is faced with the problem of beam coupling among the writing beams. Beam coupling, in basic holography, is an unwanted effect because in thick crystals it can alter considerably the value of the modulation depth $m$ along the direction of propagation of the light and, hence, the overall diffraction efficiency of the volume hologram. ${ }^{16}$ One way to limit this effect is to make the scale of variation of the light intensity $K$ small, thereby reducing the diffusive out-of-phase component of the hologram grating and applying an external voltage. Because TWM is at maximum approximately when $K$ $\cong k_{\mathrm{Db}}$ (see, e.g., Ref. 3, Chapter 4 ), in a basic holographic setup $K \ll k_{\mathrm{Db}}$ is chosen. Furthermore, in the case of $\mathrm{BaTiO}_{3}$ (in which $r_{13} \ll r_{33}$ ), one can write the hologram with ordinary polarized beams and read it with an extraordinary beam. This enables one to limit drastically the effects of TWM, thereby maintaining strong diffraction efficiencies in the read-out phase. If we were now to consider the expression given in Eq. (15) (as is done, for example, in Ref. 6), it would seem that an external voltage would enhance diffraction efficiency without modifying TWM coupling. In effect, in this regime, where conditions (17) are satisfied, we should adopt Eq. (20), from which it is apparent that enhancement by external voltage does enhance TWM coupling by means of the term that couples drift and diffusion mechanisms.

In Figs. 2(a) and 2(b) are reported, respectively, the experimental results of first- and second-harmonic Bragg diffraction enhancement of the $\mathrm{He}-\mathrm{Ne}$ beam, with $m_{1}$ $\cong 0.3, \quad \chi \cong 0.1, \quad K \cong 1.5 \times 10^{6} \mathrm{~m}^{-1}, \quad$ and $\quad k_{\mathrm{Db}} \cong 1.1$ $\times 10^{7} \mathrm{~m}^{-1}$, as measured from the angular dependence of low $m$ diffusive TWM coupling, and, finally, $l_{b}=l$. We have superimposed onto the experimental plots the theoretical fit obtained by our taking the first and second harmonics of Eq. (20) and by using Eq. (28), while neglecting the third term on the right-hand side of Eq. (20), which is of higher order in our approach as regards to Bragg diffraction efficiency (making the dependence of all diffraction orders quadratic in the external applied voltage). In this case the smallness parameter is $\epsilon \cong 0.4$. The data is obtained by use of ordinarily polarized writing beams and an extraordinarily polarized reading beam. As expected, no apparent TWM effects were observed. We obtained curve fits by varying two parameters, $m$ and $r_{33}$, taking $m \cong 0.27$ and $r_{33}=80 \mathrm{pm} / \mathrm{V}$. The value for $r_{33}$ is lower than the value independently measured $(\cong 110 \mathrm{pm} / \mathrm{V}$ at $515 \mathrm{~nm}$, even though here the wavelength is $633 \mathrm{~nm}$ ).

To assess the influence of TWM on diffraction efficiency, we performed the same experiment with extraordinary polarized writing beams, again with $m \cong 0.3$. Zero-voltage first-harmonic diffraction was measured to obtain $\eta_{1} \cong 0.015$. This indicates a diminished diffraction with respect to the above case when TWM can be neglected $\left(\eta_{1} \cong 0.020\right)$. Expected diffraction in this case (by the expressions given in Ref. 16) is $\eta_{1} \cong 0.017$.

Under these same conditions, but with extraordinary writing beams, TWM coupling was monitored for $m_{1}$ $\cong 0.91, m_{1} \cong 0.96$, and the results are shown, respectively, in Figs. 3(a) and 3(b), along with the theoretical fits obtained by our taking the first harmonic of Eq. (20) and using Eq. (29). We obtained fits by letting the modulation depth $m$ vary. The fitting values for $m_{1}$ in the two curves were, respectively, $m_{1} \cong 0.84, m_{1} \cong 0.92$. Apart from this discrepancy, these results cannot be explained (even qualitatively) by our relying solely on Eq. (15). The gain ratio value at no applied voltage is determined by the material absorption (constant for both cases) and the zero voltage TWM that is due to the diffusion-driven space-charge field (relatively strong in $\mathrm{BaTiO}_{3}$ ), which is dependent on $m$ through Eqs. (20), (29) and (30). Qualitatively, a higher $m$ corresponds to a higher zero-appliedvoltage TWM gain.

\section{Effects of Beam Size}

One of the features of the approach described in the previous Section is that it can take into account beams with finite dimension with respect to the crystal. One consequence of beam finiteness is contained in Eq. (21), in which it is apparent that the effective field in the illuminated area changes with the dimensions of this area. Intuitively this is quite obvious, in that a change in the size of the illuminated area modifies the overall resistivity of the crystal and therefore the value of the current density, thereby changing the effective field induced by an exter-

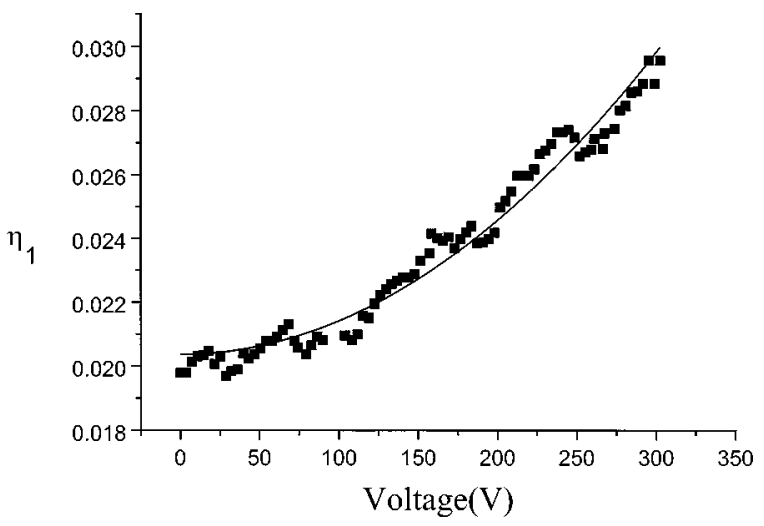

(a)

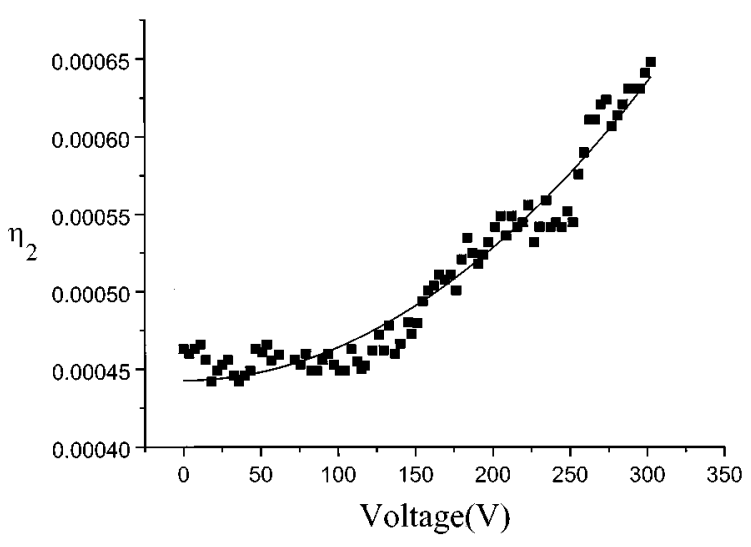

(b)

Fig. 2. Measured values of first harmonic Bragg diffraction efficiency for an expected $m_{1}=0.3$ with $\mathrm{He}-\mathrm{Ne}$, as a function of external bias voltage. The fit is obtained by taking of the first harmonic of Eq. (20) and use of relation (28). (b) Measured values of second harmonic Bragg diffraction efficiency. The fit is obtained by taking of the second harmonic of Eq. (20). 
nally applied voltage. In Fig. 4 we report the results of the TWM-coupling-enhancement factor $\sigma=g^{2} /$ $\left(V / l E_{\mathrm{Db}}\right)^{2}$ (for $\left.m_{1} \cong 0.2\right)$ for various values of $l_{b} / l$. On this plot is also superimposed the theoretical fit of Eqs. (20) and (21), in the same first-harmonic TWM approximation used in the previous paragraph. The quenching of the effective field in the illuminated area is qualitatively evident. To fit the results we must take $Q_{0}$

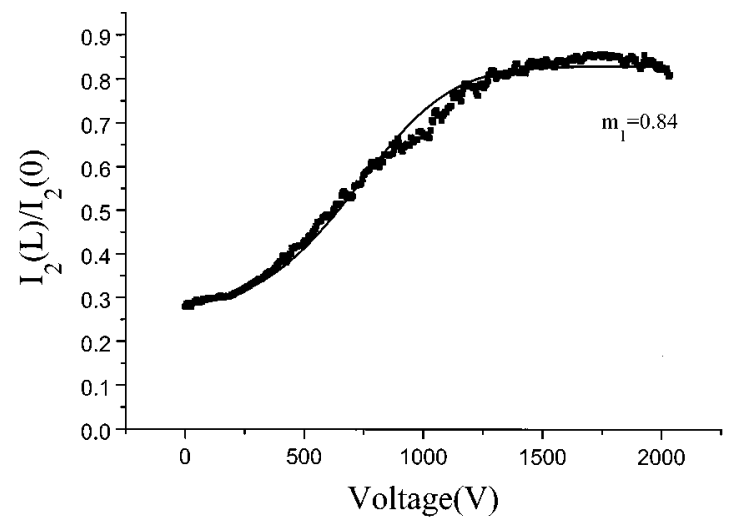

(a)

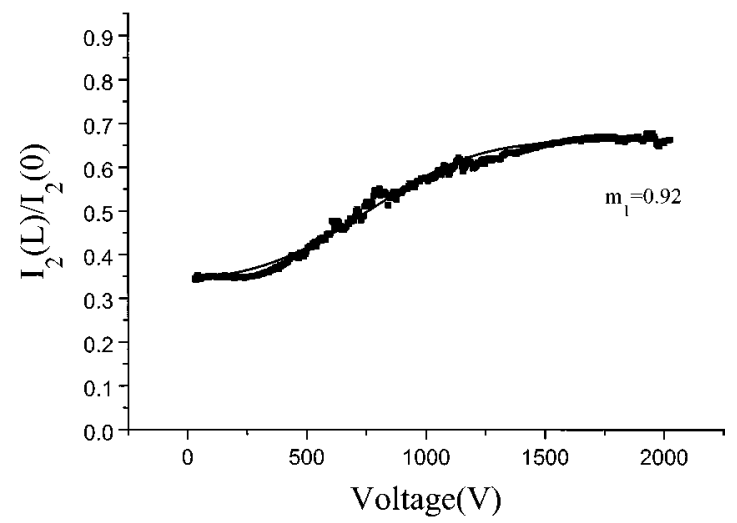

(b)

Fig. 3. (a) Measured values of TWM gain ratio of the amplified beam for $m_{1}=0.84$. Superimposed is the fitting curve obtained by taking of the first harmonic of Eq. (20) and use of Eq. (29). (b) TWM gain ratio for $m_{1}=0.92$.

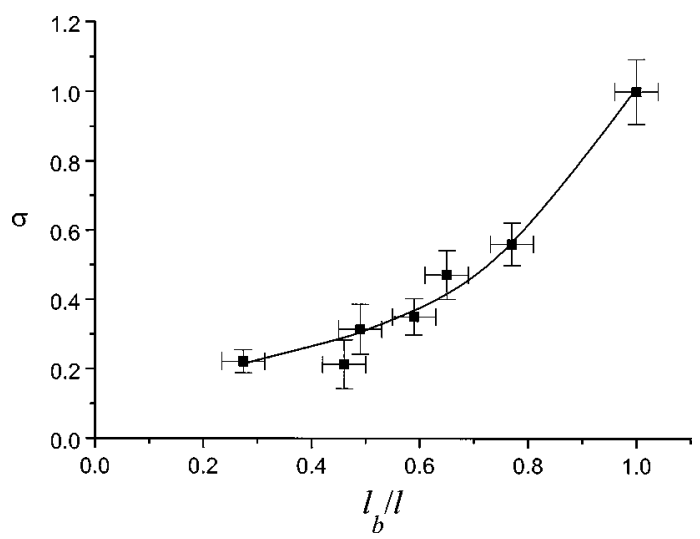

Fig. 4. Measured values of $\sigma=g^{2} /\left(V / l E_{\mathrm{Db}}\right)^{2}$ for various values of $l_{b} / l$. The fitting curve is obtained for $m_{1} \cong 0.2$ and $Q_{0}$ $\cong 1.7$. Beam coupling higher than first-harmonic is neglected. $\cong 1.7$, although this value must be viewed as an effective $Q_{0}$ because beam fanning effects, primarily responsible for the high value of $I_{b}$, generally depend on the size of the illuminated crystal region.

\section{CONCLUSIONS}

In this paper we have developed a new approach to the problem of the theoretical description of $\mathrm{PR}$ space-charge field formation in a standard TWM configuration, by exploiting a formalism recently established for the description of spatial PR solitons. This allows us to clarify the standard theory and to point out a new approximation scheme involving a single well-defined smallness parameter. In the frame of our formalism we have investigated the dependence of the electric field on the spatial extension of the interfering beams in the presence of an external voltage and have predicted TWM coupling enhancement in the high-modulation-depth regime.

To test these two last predictions, we have performed an experiment in a standard TWM configuration in $\mathrm{BaTiO}_{3}$. The experimental results are in good agreement with the theory, even in regions in which the conditions for the validity of our approximation scheme are marginally fulfilled, a circumstance that encourages us to look for an improvement in the theory by relaxing some of our more restrictive approximations.

\section{APPENDIX A}

To prove that conditions (17) and (18) imply condition (16), we begin by maximizing $\left|Y^{(0)^{\prime}}\right|$ by use of Eq. (12) for $Q$,

$$
Y^{(0)^{\prime}}=-\frac{Q^{\prime \prime}}{Q}+\left(\frac{Q^{\prime}}{Q}\right)^{2}-Y_{\mathrm{dr}}\left(\frac{Q^{\prime}}{Q}\right)
$$

and the triangular inequality,

$$
Y^{(0)^{\prime}} \leqslant\left|\frac{Q^{\prime \prime}}{\boldsymbol{Q}}\right|+\left(\frac{Q^{\prime}}{\boldsymbol{Q}}\right)^{2}+\left|Y_{\mathrm{dr}}\right|\left|\frac{Q^{\prime}}{\boldsymbol{Q}}\right| .
$$

Condition (A2) can be rewritten as

$$
Y^{(0)^{\prime}} \leqslant \frac{m_{1} \chi^{2}}{1-m_{1}}+\frac{m_{1}^{2} \chi^{2}}{\left(1-m_{1}\right)^{2}}+\left|Y_{\mathrm{dr}}\right| \frac{m_{1} \chi}{1-m_{1}},
$$

where each term in condition (A3) is greater than the corresponding term in inequality (A2). If $m_{1}<1 / 2$, then $m_{1} /\left(1-m_{1}\right)<1$, so that, using condition (A3), we have $\left|Y^{(0)^{\prime}}\right| \ll 1$ if $\chi \ll 1$ and $\left|Y_{\mathrm{dr}}\right| \chi \ll 1$, proving that one should choose $\epsilon=\chi$.

If $m_{1}>1 / 2$, then $m_{1} /\left(1-m_{1}\right)>1$, requiring $m_{1} \chi /$ $\left(1-m_{1}\right) \ll 1$ and $\left|Y_{\mathrm{dr}}\right| \ll\left(1-m_{1}\right) /\left(m_{1} \chi\right)$ (making $\chi$ $\ll 1$ as well) we have $\left|Y^{(0)^{\prime}}\right| \ll 1$, proving that one should choose $\epsilon=m_{1} \chi /\left(1-m_{1}\right)$.

As far as condition (16b) is concerned, we proceed in a similar fashion, obtaining 


$$
\begin{aligned}
\left|Y^{(0)^{\prime \prime}}\right| \leqslant & \frac{m_{1} \chi^{3}}{\left(1-m_{1}\right)}+\frac{3 m_{1}^{2} \chi^{3}}{\left(1-m_{1}\right)^{2}}+2\left(\frac{m_{1} \chi}{\left(1-m_{1}\right)}\right)^{3} \\
& +\left|Y_{\mathrm{dr}}\right| \frac{m_{1} \chi^{2}}{\left(1-m_{1}\right)}+2\left|Y_{\mathrm{dr}}\right|\left(\frac{m_{1} \chi}{\left(1-m_{1}\right)}\right)^{2}
\end{aligned}
$$

From expression (A4) it is clear that in the diffusion regime, with $g=0$, conditions (17) and (18) automatically imply condition (16b). In the drift regime the leading terms on the right-hand side are the last two. Thus we must prove that

$$
\begin{aligned}
\left|Y_{\mathrm{dr}}\right| \frac{m_{1} \chi^{2}}{\left(1-m_{1}\right)}+2\left|Y_{\mathrm{dr}}\right| & \left(\frac{m_{1} \chi}{\left(1-m_{1}\right)}\right)^{2} \\
& \ll\left|-\frac{Q^{\prime}}{Q}+\frac{g}{Q}+\frac{g}{Q} Y^{(0)^{\prime}}\right| .
\end{aligned}
$$

In this regime, according to condition (16a), it is sufficient to prove that

$$
\left|Y_{\mathrm{dr}}\right| \frac{m_{1} \chi^{2}}{\left(1-m_{1}\right)}+2\left|Y_{\mathrm{dr}}\right|\left(\frac{m_{1} \chi}{\left(1-m_{1}\right)}\right)^{2} \ll\left|Y_{\mathrm{dr}}\right|,
$$

which is implied, again, by conditions (17) and (18).

\section{APPENDIX B}

Condition (6) can be rewritten in dimensionless form as

$$
-\frac{k_{\mathrm{Db}}}{E_{\mathrm{Db}}} V=\int_{-l k_{\mathrm{Db}} / 2}^{l k_{\mathrm{Db}} / 2} Y \mathrm{~d} \xi .
$$

Only the symmetrical components of $Y$ contribute to the integral, so that it becomes, for Eqs. (15), (19) and (20),

$$
\begin{aligned}
-\frac{k_{\mathrm{Db}}}{E_{\mathrm{Db}}} V & =\int_{-l k_{\mathrm{Db}} / 2}^{l k_{\mathrm{Db}} / 2} \frac{g}{Q} \mathrm{~d} \xi \\
& =\int_{-l k_{\mathrm{Db}} / 2}^{l k_{\mathrm{Db}} / 2} \frac{g}{\left(1+Q_{0}\right)\left[1+m_{1} \cos (\chi \xi)\right]} \mathrm{d} \xi .
\end{aligned}
$$

We now approximate the function $Q_{0}$, which contains a slowly varying beam profile factor, with an appropriate constant value in a region of size $l_{b}$ (the approximate size of the beam) and zero elsewhere. The integral becomes

$$
\begin{aligned}
\int_{-l k_{\mathrm{Db}} / 2}^{l k_{\mathrm{Db}} / 2} & \frac{g}{\left(1+Q_{0}\right)\left[1+m_{1} \cos (\chi \xi)\right]} \mathrm{d} \xi \\
= & \left(l-l_{b}\right) g k_{\mathrm{Db}} \\
& +\frac{g}{\left(1+Q_{0}\right)} \int_{-l k_{\mathrm{Db}} / 2}^{l k_{\mathrm{Db}} / 2} \frac{1}{\left[1+m_{1} \cos (\chi \xi)\right]} \mathrm{d} \xi .
\end{aligned}
$$

The last integral can be approximated, for the typical case of $K l_{b} \gg 1$, by

$$
\int_{-l k_{\mathrm{Db}} / 2}^{l k_{\mathrm{Db}} / 2} \frac{1}{\left[1+m_{1} \cos (\chi \xi)\right]} \mathrm{d} \xi \cong \frac{l_{b} k_{\mathrm{Db}}}{\left(1-m_{1}^{2}\right)^{1 / 2}} .
$$

Finally, substituting Eq. (B4) into Eq. (B3), in turn substituting Eq. (B3) into Eq. (B2), and solving for $g$, we obtain Eq. (21).

\section{ACKNOWLEDGMENTS}

The work of M. Tamburrini was carried out in the framework of an agreement between Fondazione Ugo Bordoni and the Italian Communications Administration. B. Crosignani gratefully acknowledges the support of collaborative NATO research grant 930240.

M. Tamburrini can be reached by telephone, 39-6-54802230, and e-mail, tambur@fub.it.

\section{REFERENCES AND NOTES}

1. N. V. Kukhtarev, V. B. Markov, S. G. Odulov, M. S. Soskin, and V. L. Vinetski, Ferroelectrics 22, 961 (1979).

2. P. Günter and J. P. Huignard, eds., Photorefractive Materials and Their Applications I (Springer-Verlag, Berlin, 1988); Photorefractive Materials and Their Applications II (Springer-Verlag, Berlin, 1989).

3. P. Yeh, Introduction to Photorefractive Nonlinear Optics (Wiley, New York, 1993).

4. L. Solymar, D. J. Webb, and A. Grunnet-Jepsen, The Physics and Applications of Photorefractive Materials (Clarendon, Oxford, UK, 1996).

5. L. B. Au and L. Solymar, Opt. Lett. 13, 660 (1988).

6. M. G. Moharam, T. K. Gaylord, R. Magnusson, and L. Young, J. Appl. Phys. 50, 5642 (1979).

7. N. V. Kukhtarev, P. Buchhave, and S. F. Lyuksyutov, Phys. Rev. A 55, 3133 (1997).

8. Y. H. Lee and R. W. Hellwarth, J. Appl. Phys. 71, 916 (1992).

9. A. Bledowski, J. Otten, and K. H. Ringhofer, Opt. Lett. 16, 672 (1991)

10. M. Segev, G. C. Valley, B. Crosignani, P. Di Porto, and A. Yariv, Phys. Rev. Lett. 73, 3211 (1994).

11. M. Segev, M. Shih, and G. C. Valley, J. Opt. Soc. Am. B 13, 706 (1996).

12. B. Crosignani, P. Di Porto, A. Degasperis, M. Segev, and S. Trillo, J. Opt. Soc. Am. B 14, 3078-3090 (1997).

13. F. Vachss and L. Hesselink, J. Opt. Soc. Am. A 5, 690 (1988).

14. Conditions (17) express in a compact form the limitations imposed by our resolving scheme. The first is quite restrictive. Apart from the possibility of being relaxed at the cost of greater algebraic complexity, it has the advantage of being valid for all values of $0<m_{1}<1$. The condition on the drift field is related to the so-called saturation field and is easily verified in most doped crystals for standard applied voltages.

15. A. Yariv, Optical Electronics, 5th ed. (Wiley, New York, 1995).

16. J. H. Hong and R. Saxena, Opt. Lett. 16, 180 (1991). 